\title{
Relationship of superior mesenteric artery thrombus density with transmural intestinal necrosis on multidetector computed tomography in acute mesenteric ischemia
}

\author{
Wei Tang ${ }^{1,2}$, Jing Zhang ${ }^{1 \wedge}$, Lian-Qin Kuang ${ }^{1 \wedge}$, Kun-Ming Yi ${ }^{1}$, Chun-Xue Li $^{3} \wedge$, Yi Wang ${ }^{1}$ \\ ${ }^{1}$ Department of Radiology, Daping Hospital, Army Medical University, Chongqing, China; ${ }^{2}$ Department of Radiology, Affiliated Hospital of North \\ Sichuan Medical College, Nanchong, China; ${ }^{3}$ Department of Gastrointestinal Surgery, Daping Hospital, Army Medical University, Chongqing, \\ China
}

Correspondence to: Yi Wang. Department of Radiology, Daping Hospital, Army Medical University, No. 10 Changjiang Branch Road, Yuzhong District, Chongqing 400042, China. Email: ywhxl@qq.com.

Background: Acute arterial occlusive mesenteric ischemia with transmural intestinal necrosis (TIN) is a fatal disease, which is difficult to diagnose on multidetector computed tomography (MDCT). The aim of the present study was to determine the relationship of superior mesenteric artery (SMA) thrombus density with TIN on MDCT in patients with acute mesenteric ischemia (AMI) due to SMA thromboembolism.

Methods: In this retrospective study, 33 patients who underwent abdominal MDCT and angiography for AMI due to SMA thromboembolism were divided into two groups: the AMI with TIN group and the AMI without TIN group. We analyzed the relationships of clinical characteristics, qualitative MDCT signs, and SMA thrombus density with TIN. The SMA thrombus density was measured on non-contrast MDCT. Univariate and multivariate analyses were performed to determine the risk factors for predicting TIN. The diagnostic performances of risk factors were evaluated by receiver-operating characteristic (ROC) curve analysis.

Results: Of the patients with AMI enrolled in this study, 33.3\% (11/33) were diagnosed with TIN. Peritonitis $(\mathrm{P}=0.042)$, bowel wall thinning $(\mathrm{P}=0.033)$, and pneumatosis/portomesenteric gas $(\mathrm{P}=0.010)$ were significantly associated with TIN. AMI patients with TIN exhibited a higher SMA thrombus density than AMI patients without TIN [41.2 \pm 6.1 vs. 34.2 \pm 3.0 Hounsfield unit $(\mathrm{HU}), \mathrm{P}=0.003]$. Multivariate analysis showed that SMA thrombus density was an independent predictor of TIN [P=0.044, hazard ratio (HR): 1.82, 95\% confidence interval (CI): 1.02-3.25]. For diagnosing AMI with TIN, the area under the ROC curve (AUC) of SMA thrombus density (0.83) was larger than those of peritonitis (0.68), bowel wall thinning (0.66), and pneumatosis/portomesenteric gas (0.71).

Conclusions: In patients with AMI, erythrocyte-rich thrombus blocking the SMA trunk which has a higher density on MDCT is prone to the occurrence of TIN compared with erythrocyte-scarce thrombus with a lower density. SMA thrombus density could be an independent risk factor for TIN in patients with AMI due to SMA thromboembolism.

Keywords: Superior mesenteric artery (SMA); mesenteric ischemia; thromboembolism; multidetector computed tomography (MDCT)

Submitted Apr 22, 2020. Accepted for publication Mar 12, 2021.

doi: 10.21037/qims-20-604

View this article at: http://dx.doi.org/10.21037/qims-20-604

^ ORCID: Jing Zhang, 0000-0002-6409-6537; Lian-Qin Kuang, 0000-0002-2774-9818; Chun-Xue Li, 0000-0002-3365-6773. 


\section{Introduction}

Superior mesenteric artery (SMA) thromboembolism is the most common cause of acute mesenteric ischemia (AMI), and its etiology can be classified as thrombosis and embolism (1). The development of SMA thrombosis is superimposed on pre-existing severe atherosclerotic disease, and the majority of SMA embolic events are thromboembolic in nature and arise from a cardiac source (2). SMA thrombus consists of platelets, fibrin, and erythrocytes in variable proportions (3). Berndt et al. reported that tightly packed, erythrocyte-rich thrombus showed low perviousness, which could impede blood and contrast agent penetration in patients with stroke (4). Conversely, platelet/fibrin-rich thrombus appears to allow the blood and contrast agent to penetrate the clot more easily. From the results of Berndt et al.'s study, we deduced that the relative erythrocyte and platelet content of SMA thrombus may contribute to insufficient blood supply to the intestines and ischemia-reperfusion injury in patients with AMI (4). Blood supply insufficiency and ischemiareperfusion injury are the main factors contributing to intestinal ischemic injury (5).

Among patients with AMI, the severity of intestinal ischemic injury is closely related to mortality, especially for those with arterial occlusion. The prevalence of arterial occlusion is higher among elderly than young patients with AMI, as older patients often have comorbidities and are prone to the occurrence of intestinal ischemic necrosis $(1,6)$. The development and extent of intestinal necrosis often leads to septic shock, multiple organ function failure, and even death (7). Therefore, the identification of patients with irreversible intestinal ischemic injury who will not benefit from long-term conservative treatment and will require timely intestinal resection due to transmural necrosis forms the cornerstone of livesaving strategies for AMI (8).

Multidetector computed tomography (MDCT) is recommended as the first-line modality for the evaluation of AMI (9-11). However, identifying patients with AMI who require intestinal resection remains a challenge, which emphasizes the need to determine accurate predictive factors for transmural intestinal necrosis (TIN) requiring resection (12). We presume that there is a relationship between the composition of SMA thrombus and intestinal necrosis in AMI. Non-contrast MDCT and MDCT angiography can detect thrombus in large arteries and provide information about its composition based on Hounsfield units (HU) $(13,14)$. A platelet-rich thrombus contains varying amounts of atheromatous and cellular debris, fibrin, and platelets, but have few red cells; thus, it has a lower HU value. In contrast, an erythrocyte-rich thrombus contains higher concentrations of hemoglobin, so it has a higher HU value (15). The aim of the present study was to investigate whether a relationship exists between SMA thrombus density and irreversible TIN on MDCT in patients with AMI due to SMA thromboembolism.

\section{Methods}

\section{Patient population}

The study protocol was approved by the institutional review board of Daping Hospital, which waived the requirement to obtain informed consent from all patients due to the study's retrospective nature. We retrospectively enrolled patients with clinically verified or suspected AMI who were referred to our department for MDCT examination between February 2013 and December 2019. Prior to MDCT scanning, all patients were clinically examined by either a gastroenterologist or a vascular surgeon. The inclusion criteria were as follows: (I) patients with AMI due to SMA thromboembolism; and (II) the time interval between MDCT examination and surgery was less than 24 hours. The exclusion criteria were as follows: (I) the time interval between MDCT examination and surgery was more than 24 hours; and (II) patients with venous mesenteric ischemia, non-occlusive mesenteric ischemia, vasculitis, dissecting aneurysm, or bowel obstruction-induced mesenteric ischemia. Finally, 40 patients with AMI caused by SMA thromboembolism who met the inclusion criteria were included in the present study.

\section{MDCT scanning}

A 64-row MDCT scanner (LightSpeed VCT 64; GE Healthcare, Milwaukee, WI, USA) or a 256-slice MDCT scanner (Brilliance iCT 256; Philips Healthcare, Cleveland, $\mathrm{OH}$, USA) was used to perform abdominal unenhanced and dual-phase enhanced (hepatic arterial and portal venous phases) imaging. The parameters were set as follows: collimation of $64 \times 0.625 \mathrm{~mm}$ or $256 \times 0.5 \mathrm{~mm}$, table speed of 64 or $256 \mathrm{~mm}$ per rotation, pitch 0.984 , matrix $512 \times 512$, field of view $240-400 \mathrm{~mm}$, tube voltage $120 \mathrm{kV}$, and tube current 250-300 mA. The scanning coverage extended from the dome of the diaphragm to the pubic symphysis. 
Non-ionic iodinated contrast agent with an iodine concentration of $370 \mathrm{mg} / \mathrm{mL}$ (Ultravist; Bayer Schering Pharma, Berlin, Germany) was injected intravenously via a power injector at a rate of $4 \mathrm{~mL} / \mathrm{s}$, with a dose of $2 \mathrm{~mL} / \mathrm{kg}$ body weight and the upper dose limit set to $120 \mathrm{~mL}$ for each patient. Images were acquired in the hepatic arterial and portal venous phases at 20 and 50 seconds after the completion of the contrast agent administration, respectively.

\section{Image analysis}

All data were transferred to an offline workstation (ADW 4.3; GE Healthcare, Milwaukee, WI, USA) for image postprocessing and analysis. Multiplanar reformation, curved planar reformation, volume rendering, and maximum intensity projection were used to reformat images and analyze the signs of intestinal ischemic injury, the location and extent of SMA thromboembolism, the involvement of SMA branches, and the severity of SMA stenosis. The images were independently reviewed by two abdominal radiologists each with more than 10 years of experience, and final agreement was achieved by consensus.

The SMA trunk can be classified into the following three zones: the SMA trunk proximal to the $1^{\text {st }}$ major branch (zone 1); the SMA trunk between the inferior pancreaticoduodenal artery and middle colic artery (zone 2); and the SMA trunk distal to the middle colic artery (zone 3) (16). As thrombus of different lengths may extend to more than 1 zone of the SMA trunk, the findings of SMA thromboembolism on MDCT were described as 1 zone of the SMA trunk plus another. The degree of SMA trunk stenosis was defined as complete or partial occlusion based on the enhanced axial and reformatted images.

\section{Quantification of the thrombus $H U$}

Based on the most proximal filling defect of thrombus on axial MDCT images in the arterial phase, two independent radiologists, who were blinded to the patients' clinical data, manually placed regions of interest (ROIs) in the center of the thrombus on non-contrast MDCT images, while avoiding the adjacent other structures in each section. Measurement of the attenuation of the ROIs was performed using magnified images on the same workstation above mentioned. For all ROIs, the HU values were obtained by averaging all voxels within the ROI. The final $\mathrm{HU}$ value for each thrombus was calculated by summing the HU value of the ROIs in each section of the thrombus and dividing it by the number of sections. The HU values were independently determined by two raters, and their results were averaged.

\section{Treatment and definition of irreversible TIN}

Treatment decisions were made by the attending physicians according to patients' clinical status, laboratory tests, and initial MDCT findings. Treatments included conservative anticoagulant therapy, endovascular therapy, and laparotomy.

As intestinal ischemic injury is a progressive process, patients with AMI with TIN confirmed by pathology, MDCT findings, or surgical findings, were included in the present study. Irreversible TIN was defined based on evidence of intestinal perforation on MDCT images, the discovery of extensive necrosis during open-close laparotomy procedures in unresected patients, and intestinal necrosis confirmed by pathology in patients who underwent intestinal resection. Patients with superficial and nontransmural ischemic necrosis upon pathological assessment were excluded. Patients who recovered from AMI with no need for intestinal resection after receiving conservative anticoagulant therapy and endovascular therapy were considered not to have TIN after 3 months of follow-up, as transmural necrosis could be ruled out at this point.

\section{Statistical analysis}

Categorical variables were reported as frequencies or percentages and were compared using the $\chi^{2}$-test or Fisher's exact test, as appropriate. Continuous variables were reported as means \pm standard deviations (SDs) and were compared with the independent-samples $t$-test. Variables associated with TIN were determined by univariate analysis, and variables with $\mathrm{P}<0.05$ in the univariate analysis were selected for multivariate analysis and receiver-operating characteristic (ROC) curve analysis. The results of the multivariate analysis were expressed as hazard ratios (HRs) with $95 \%$ confidence intervals (CIs) and corresponding $\mathrm{P}$ values. The Youden index (= sensitivity + specificity -1$)$ was used to determine the cutoff value of the ROC curve. Statistical analyses were performed using SPSS version 22.0 statistical software package (IBM, Armonk, NY, USA) and MedCalc software 17.2 (MedCalc Software, Mariakerke, Belgium). For all tests, $\mathrm{P}<0.05$ was considered to indicate a statistically significant difference. 


\section{Results}

\section{Patients' clinical characteristics}

Of the 40 patients with AMI caused by SMA thromboembolism, 5 patients who had calcification at the site of the thrombus were excluded due to the impact of calcification on the attenuation of thrombus on MDCT; also, 2 patients with thromboembolism only in branches of the SMA were excluded due to the difficulty in measuring the density of such thrombus on MDCT. Finally, 33 patients (including 13 females and 20 males) with a mean age of $69.4 \pm 11.8$ (range, 43-87) years were included for analysis in the present study.

Bowel resection was performed in 12 (36.4\%) of the 33 patients. Among these 12 patients, 5 underwent bowel resection following endovascular therapy, due to suspected intestinal necrosis, and 7 received laparotomy for both SMA recanalization and bowel resection. Pathological analysis of the surgical specimens following resection confirmed TIN in 10 of the 12 patients who received bowel resection, while superficial ischemic injury was confirmed in the other 2 patients. Of the 33 study participants, 1 (3\%) patient died a few hours after an open-close laparotomy procedure without resection from extensive bowel infarction. The other 20 patients $(60.6 \%)$ were considered to have reversible intestinal ischemic injury and recovered with no need for bowel resection. Among these 20 patients, 14 received endovascular therapy (Figure 1). Intraoperative recanalization was performed in 2 patients, while 4 patients underwent conservative treatment (Figure 2). Eventually, TIN and the absence of intestinal necrosis were the final diagnoses for 11 $(33.3 \%)$ and $22(66.7 \%)$ patients, respectively (Table 1).

Table 1 summarizes the clinical characteristics of the 33 patients. There was no significant difference between AMI patients with and without TIN in terms of age, sex, risk factors of thrombus formed, history of cardiovascular disease, or mean symptom duration prior to admission. Regarding clinical presentations, there was a significant difference between AMI patients with and without TIN in terms of the incidence of peritonitis $(54.5 \%$ vs. $18.2 \%$, $\mathrm{P}=0.042$ vs. $\mathrm{P}<0.05)$. However, for other clinical symptoms, there was no significant difference between AMI patients with and without TIN $(\mathrm{P}>0.05)$ (Table 1).

\section{MDCT findings and SMA thrombus density were associated with TIN}

The MDCT findings and SMA thrombus density in the
33 AMI patients are summarized in Table 2. There were significant differences between AMI patients with and without TIN in terms of the incidence of bowel wall thinning $(36.4 \%$ vs. $4.5 \%, \mathrm{P}=0.033)$ and pneumatosis/ portomesenteric gas $(45.5 \%$ vs. $4.5 \%, \mathrm{P}=0.010$ ) (Figure 3). No significant difference was found between AMI patients with and without TIN for other MDCT findings of AMI $(\mathrm{P}>0.05)$. Compared with AMI patients without TIN, those with TIN had a higher SMA thrombus density $(41.2 \pm 6.1$ vs. 34.2 $\pm 3.0, \mathrm{P}=0.003$ ) (Figures 2,3; Table 2). Multivariate analysis showed that SMA thrombus density measured on MDCT could be an independent risk factor for predicting TIN in patients with AMI (HR: 1.18, 95\% CI: 1.02-3.25, $\mathrm{P}=0.044$ ); however, peritonitis, bowel wall thinning, and pneumatosis/portomesenteric gas were not found to be independent risk factors for predicting TIN in patients with AMI $(\mathrm{P}>0.05)$ (Table 3).

\section{ROC curve analysis of variables associated with TIN}

The areas under the ROC curves (AUCs) of peritonitis, bowel wall thinning, pneumatosis/portomesenteric gas, and SMA thrombus density for diagnosing TIN in patients with AMI were $0.68,0.66,0.71$, and 0.83 , respectively (Table 4; Figure 4). SMA thrombus density had a larger AUC than the other 3 variables. The sensitivity and specificity of peritonitis, bowel wall thinning, pneumatosis/ portomesenteric gas, and SMA thrombus density in the diagnosis of TIN in patients with AMI were $54.5 \%$ and $81.8 \%, 36.4 \%$ and $95.5 \%, 45.5 \%$ and $95.5 \%$, and $72.7 \%$ and $86.4 \%$ (cutoff value: $36.2 \mathrm{HU}$ ), respectively (Table 4; Figure 5).

\section{Discussion}

Despite the lack of any early, specific biomarkers for the diagnosis of intestinal necrosis, diagnostic accuracy has been improved by MDCT (17). Once the diagnosis of AMI has been established on MDCT by radiologists and surgeons, it is important to discriminate AMI patients with intestinal necrosis from those without intestinal necrosis (18-20). Previous studies have indicated that some MDCT signs are associated with intestinal necrosis in patients with AMI $(12,21,22)$. Nuzzo et al. reported that bowel loop dilation was associated with TIN in arterial, venous, and nonocclusive AMI (12), and Calame et al. reported that TIN in occlusive AMI has distinct CT findings, characterized by decreased or absent bowel wall enhancement and less 

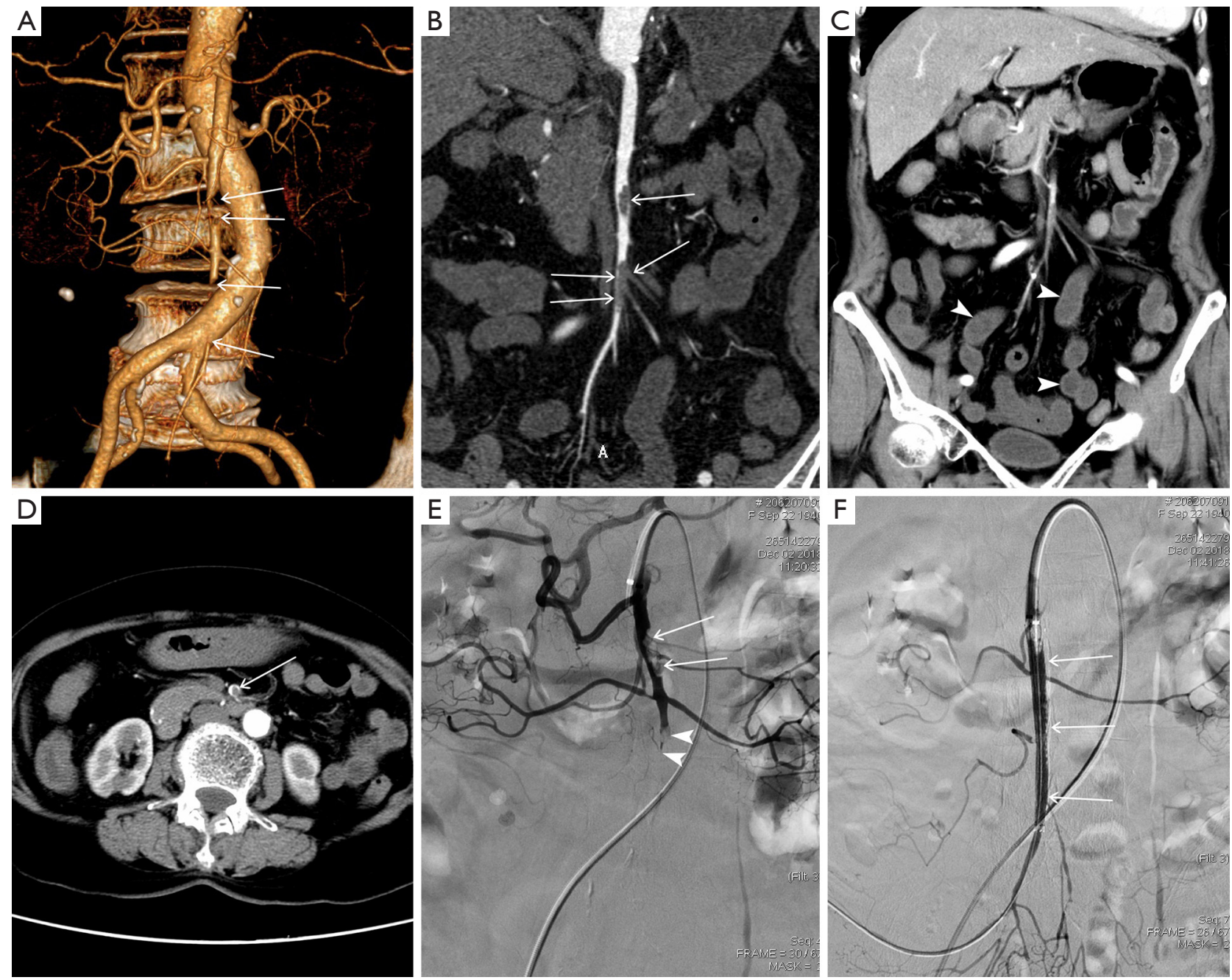

Figure 1 A 78-year-old female patient with acute mesenteric ischemia (AMI) due to superior mesenteric artery (SMA) thromboembolism. Multidetector computed tomography (MDCT) with volume rendering (A) and curved planar reformation (B) images showed a thrombus in the trunk and branches of the SMA (arrows). Coronal (C) and axial (D) images showed a slight decrease in small bowel wall enhancement (arrowheads) and a low-density filling defect in the SMA lumen (arrow). Digital subtraction angiography (E) showed that the SMA trunk had a filling defect with severe stenosis (arrows), and its distal branches were completely occluded (arrowheads). After thrombolysis treatment and the placement of a bare-metal stent (arrows), catheter angiogram (F) showed that the SMA trunk and its branches were now patent. Finally, the patient underwent endovascular treatment for mesenteric recanalization and recovered from the ischemic intestinal injury.

mesenteric fat stranding (22), which is inconsistent with our findings. The reasons for this inconsistency are as follows. Our study sample included only patients with AMI due to arterial occlusion, which is different from Nuzzo et al.'s study sample, which included patients with AMI of three etiologies. Calame et al. compared the prevalence of MDCT signs in occlusive AMI with TIN, non-occlusive AMI with TIN, and strangulated small bowel obstruction (SBO) with TIN, but did not compare the prevalence of MDCT signs between occlusive AMI with and without TIN. In these previous studies, there was controversy regarding which MDCT signs were associated with TIN in AMI; these MDCT signs are qualitative indicators. As far as we know, little research has been conducted on quantitative MDCT findings associated with TIN in patients with AMI, except for the quantification of bowel wall enhancement associated with AMI secondary to bowel obstruction (23).

Prompted by other studies that analyzed the composition 

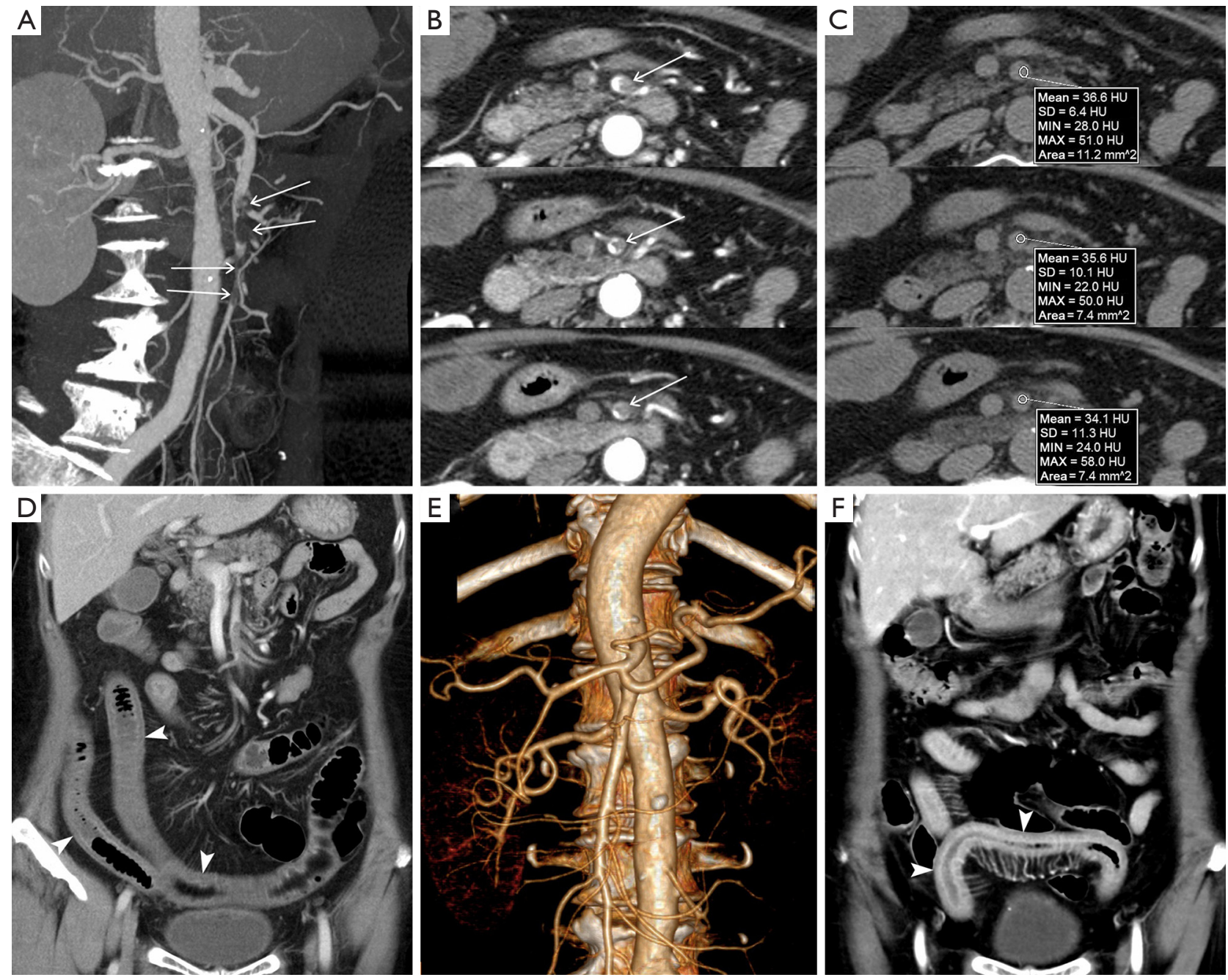

Figure 2 A 76-year-old female patient with acute mesenteric ischemia (AMI) due to superior mesenteric artery (SMA) thromboembolism. Multidetector computed tomography (MDCT) with maximum intensity projection (A) showed multiple filling defects due to thromboembolism in the SMA and its branches (arrows). Based on the most proximal filling defect (arrows) in the SMA lumen on three consecutive axial images in the arterial phase (B), the SMA thrombus density was measured on three corresponding axial non-contrast images; the Hounsfield unit (HU) values of the thrombus in these sections were 36.6, 35.6, and 34.1 respectively (C). The mean HU value (35.4) of the whole thrombus could then be calculated. Coronal image (D) showed small bowel wall edema and thickness (arrowheads). After conservative thrombolysis treatment, volume rendering (E) and coronal (F) images showed that the thrombus in the SMA trunk had disappeared, and the small bowel edema and thickness (arrowheads) had improved significantly.

or thrombus density in patients with acute ischemic stroke (4), acute myocardial infarction (24), and deep vein thrombosis (25), in the present study, we analyzed whether the SMA thrombus density on MDCT has the ability to predict intestinal necrosis in patients with AMI. Interestingly, there was a difference in SMA thrombus density between AMI patients with and without TIN; the patients with AMI are prone to the occurrence of
TIN, while a higher thrombus density blocks the SMA trunk. Furthermore, SMA thrombus density could be an independent risk factor for predicting TIN; to the best of our knowledge, this has not been directly proven in arterial occlusion due to AMI before now.

The density of a thrombus on MDCT increases with the erythrocyte percentage. Erythrocyte-rich thrombus has higher HU values than platelet-rich debris-filled 
Table 1 Clinical characteristics of 33 patients with AMI due to SMA thromboembolism stratified by the presence of TIN

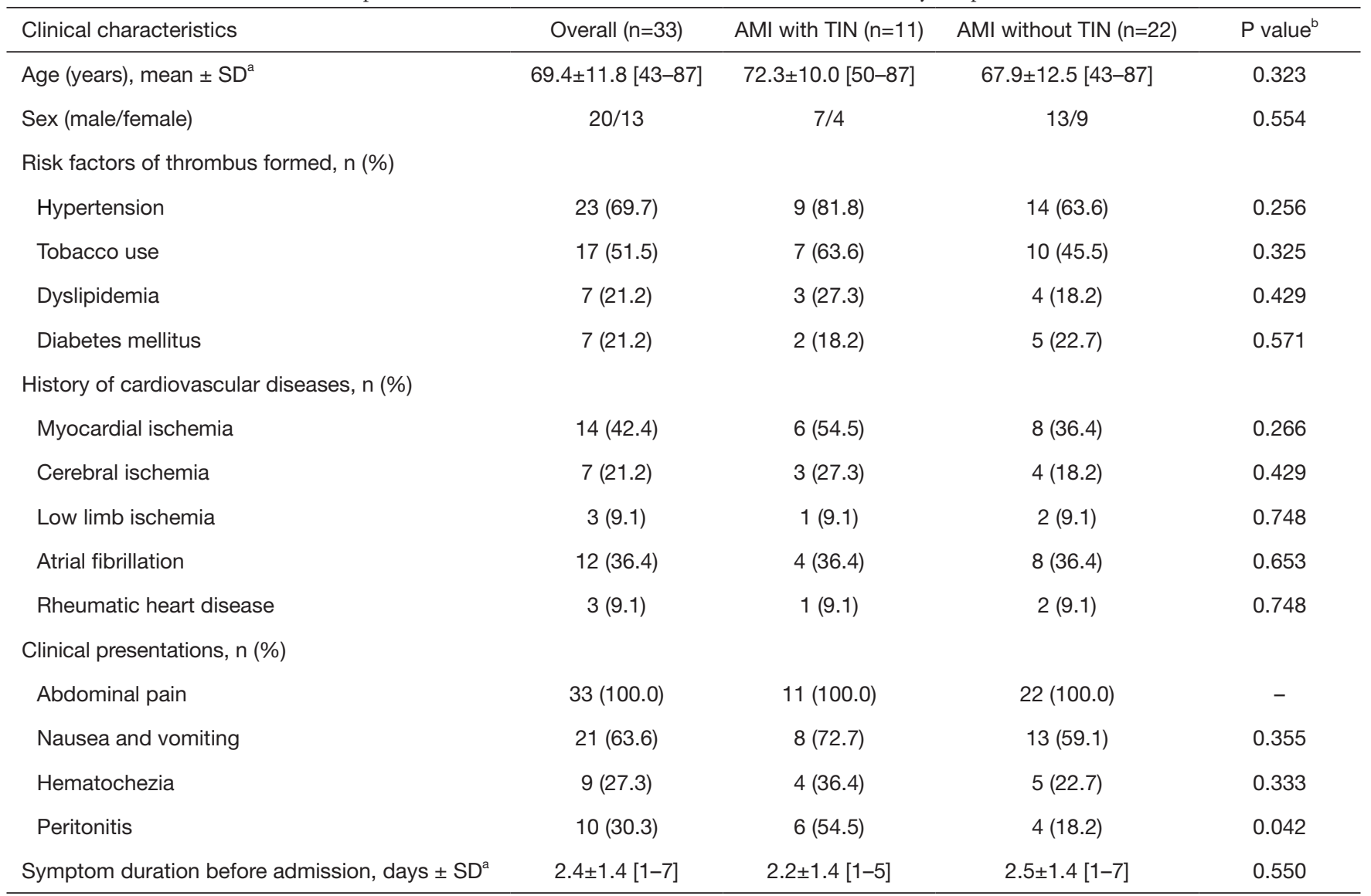

a , unless otherwise noted, data are presented as number (\%); ${ }^{b}$, comparison between AMI patients with and without TIN. AMI, acute mesenteric ischemia; SMA, superior mesenteric artery; TIN, transmural intestinal necrosis; SD, standard deviation.

thrombus, because HU values have a linear correlation with hemoglobin (15). Gersh et al. reported that the proportion and incorporation pattern of erythrocytes influence the permeability and viscoelasticity of pathological thrombus (26). In their study, van Gelder et al. reported that the addition of erythrocytes led to a decrease in the permeability of fibrin clots which was proportional to both the total surface area of the erythrocytes and their total concentration (27). Based on Gersh et al. and van Gelder et al.'s studies, erythrocyte-rich thrombus with low permeability could impede blood penetration, while erythrocyte-scarce thrombus seems to allow the blood to penetrate the clot more easily due to their high permeability. We conclude that erythrocyte-rich thrombus obstructing SMA would result in more severe intestinal ischemic injury than erythrocyte-scarce thrombus in patients with AMI.

At the beginning, thrombus of the SMA formed in situ or arising from a cardiac source are mainly composed of platelets and fibrin. However, as erythrocytes become trapped in fibrin mesh, the proportion of erythrocytes in thrombus gradually increases with the prolongation of ischemic time; consequently, the composition of thrombus includes fibrin and erythrocytes in variable proportions $(3,26,28)$. Therefore, erythrocyte-rich thrombus with higher HU values on MDCT is associated with a longer ischemic time than platelet or fibrin-rich thrombus. A longer ischemic time may lead to the occurrence of TIN in patients with AMI.

Although the mean symptom duration before admission showed no significant difference between AMI patients with and without TIN in our study, it is likely that non-specific complaints precluded our ability to record accurate time from acute onset to admission, especially in elderly AMI patients with severe comorbidities (1,6,29). Furthermore, the development of thrombus in the SMA may precede the onset of clinical presentation in some patients with AMI (30). 
Table 2 Univariate analysis of the MDCT findings and SMA thrombus density for the 33 patients with AMI due to SMA thromboembolism

\begin{tabular}{|c|c|c|c|c|}
\hline MDCT findings & Overall $(n=33)$ & AMI with TIN (n=11) & AMI without TIN ( $n=22)$ & $P$ value \\
\hline Partial occlusion & $17(51.5)$ & $4(36.4)$ & $13(59.1)$ & \\
\hline Complete occlusion & $16(48.5)$ & $7(63.6)$ & $9(40.9)$ & \\
\hline SMA branches, n (\%) & & & & 0.218 \\
\hline No involvement & $17(51.5)$ & $4(36.4)$ & $13(59.1)$ & \\
\hline Extent of SMA trunk thrombus, $\mathrm{n}(\%)$ & & & & 0.957 \\
\hline Zone 1 & $2(6.1)$ & $1(9.1)$ & $1(4.5)$ & \\
\hline Zone 2 & $5(15.2)$ & $2(18.2)$ & $3(13.6)$ & \\
\hline Zone $1+2+3$ & $5(15.1)$ & $2(18.2)$ & $3(13.6)$ & \\
\hline \multicolumn{5}{|l|}{ Signs of AMI, n (\%) } \\
\hline Bowel wall thickening & $14(42.4)$ & $4(36.4)$ & $10(45.5)$ & 0.453 \\
\hline Bowel wall edema & $3(9.1)$ & 0 & $3(13.6)$ & 0.282 \\
\hline Bowel wall thinning & $5(15.2)$ & $4(36.4)$ & $1(4.5)$ & 0.033 \\
\hline Increased bowel wall attenuation & $1(3)$ & $1(9.1)$ & 0 & 0.333 \\
\hline Decreased bowel wall enhancement & $21(63.6)$ & $6(54.5)$ & 15 (68.2) & 0.347 \\
\hline Pneumatosis/portomesenteric gas & $6(18.2)$ & $5(45.5)$ & $1(4.5)$ & 0.010 \\
\hline
\end{tabular}

${ }^{a}$, unless otherwise noted, data are presented as number (\%); ${ }^{b}$, comparison between AMI patients with and without TIN. MDCT, multidetector computed tomography; SMA, superior mesenteric artery; SD, standard deviation; AMI, acute mesenteric ischemia; TIN, transmural intestinal necrosis; HU, Hounsfield unit.

These clinical features suggest that the recorded onset time does not reflect the true ischemic time for patients with AMI. However, the density of a thrombus may be closely related to ischemic time in patients with AMI. As the process and mechanism of SMA thrombosis are complex, the time of SMA thrombosis is difficult to determine; therefore, the relationship between SMA thrombus density and ischemic time warrants further study.

In the ROC curve analysis, the AUC of SMA thrombus density $(0.83)$ was larger than those of peritonitis (0.68), bowel wall thinning (0.66), and pneumatosis/ portomesenteric gas (0.71) for diagnosing TIN in patients with AMI, which suggested that SMA thrombus density may be more discriminative than the other three variables for predicting TIN. SMA thrombus density had higher sensitivity $(72.7 \%)$ and, with the exception of peritonitis, lower specificity $(86.4 \%)$ than the other three variables for diagnosing AMI patients with TIN. Peritonitis is considered to be a common indication for surgical exploration, and patients with AMI and peritonitis may have irreversible or reversible intestinal ischemic injury (31). Thinning of the bowel wall can be caused by volume loss of the tissues and vessels in the bowel wall and by the loss of intestinal muscular tone (adynamic ileus); this is often associated 

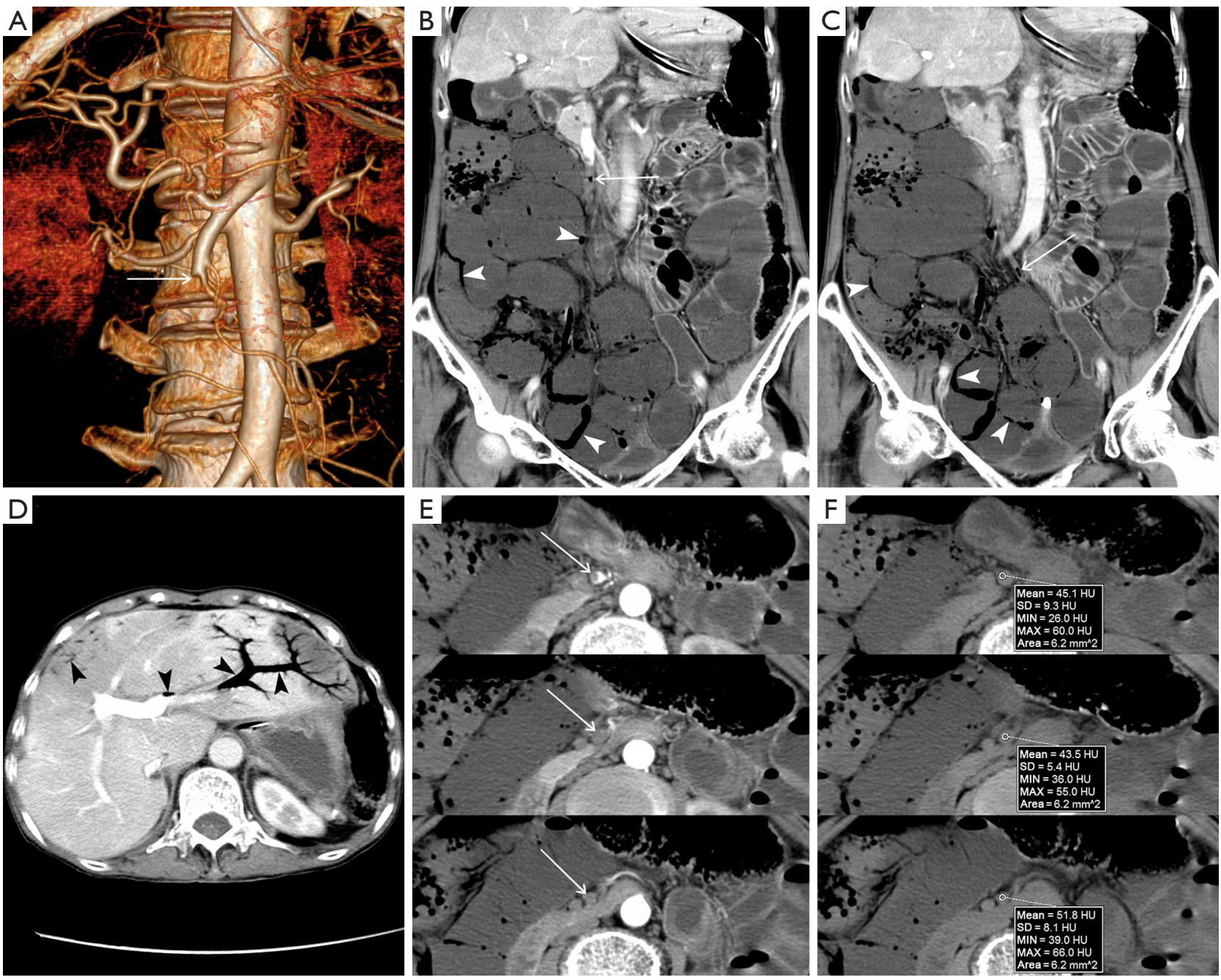

Figure 3 A 75-year-old female patient with acute mesenteric ischemia (AMI) due to superior mesenteric artery (SMA) thromboembolism. Multidetector computed tomography (MDCT) volume rendering (A) showed SMA complete occlusion (arrow) due to a thrombus; its distal branches could not be visualized. Coronal (B,C) and axial (D) images showed dilated small bowel loops with wall thinning, decreased wall enhancement, and pneumatosis (white arrowheads). Mesenteric veins in the right and lower abdomen were not filled with contrast agent, and mesenteric (arrows) and portal (black arrowheads) venous gas was also observed. Based on the most proximal filling defect (arrows) in the SMA lumen on three consecutive axial images in the arterial phase (E), the SMA thrombus density was measured on three corresponding axial non-contrast images. Hounsfield unit $(\mathrm{HU})$ values of the thrombus on these sections were 45.1, 43.5, and 51.8, respectively (F). The mean HU value (46.8) of the whole thrombus could then be calculated. Extensive transmural intestinal necrosis (TIN) was found during open-close laparotomy procedures in the patient, who eventually died.

with superficial muscular layer or transmural necrosis in arterial occlusive ischemia (32). The causes of pneumatosis/ portomesenteric gas include mucosal injury, increased intraluminal pressure, and transmural necrosis (33). The above reasons may explain why these qualitative MDCT signs had lower sensitivity than SMA thrombus density for the diagnosis of AMI patients with TIN.
The different treatments received by the patients in our study are worth mentioning. Patients with AMI require urgent management, which is challenging due to individual patient differences, the dependence on the physician's experience, and the condition's low overall incidence. Furthermore, there is a lack of type I evidence from large, randomized controlled, multicenter prospective studies to 
Table 3 Multivariate analysis of risk factors associated with TIN

\begin{tabular}{|c|c|c|c|}
\hline Risk factors & \multicolumn{3}{|c|}{ Multivariate analysis } \\
\hline Peritonitis & 0.061 & - & - \\
\hline Bowel wall thinning & 0.054 & - & - \\
\hline Pneumatosis/portomesenteric gas & 0.914 & - & - \\
\hline
\end{tabular}

TIN, transmural intestinal necrosis; HR, hazard ratio; $\mathrm{Cl}$, confidence interval; SMA, superior mesenteric artery; HU, Hounsfield unit.

Table 4 ROC curve analysis of the variables associated with TIN

\begin{tabular}{|c|c|c|c|c|}
\hline Variables & $\mathrm{AUC} \pm \mathrm{SD}(95 \% \mathrm{Cl})$ & Sensitivity (\%) & Specificity (\%) & Cutoff values \\
\hline Bowel wall thinning & $0.66 \pm 0.08(0.47-0.81)$ & 36.4 & 95.5 & - \\
\hline Pneumatosis/portomesenteric gas & $0.71 \pm 0.08(0.52-0.85)$ & 45.5 & 95.5 & - \\
\hline Density of SMA thrombus (HU) & $0.83 \pm 0.09(0.66-0.94)$ & 72.7 & 86.4 & 36.2 \\
\hline
\end{tabular}

ROC, receiver-operating characteristic; TIN, transmural intestinal necrosis; AUC, area under the ROC curve; SD, standard deviation; Cl, confidence interval; SMA, superior mesenteric artery; HU, Hounsfield unit.

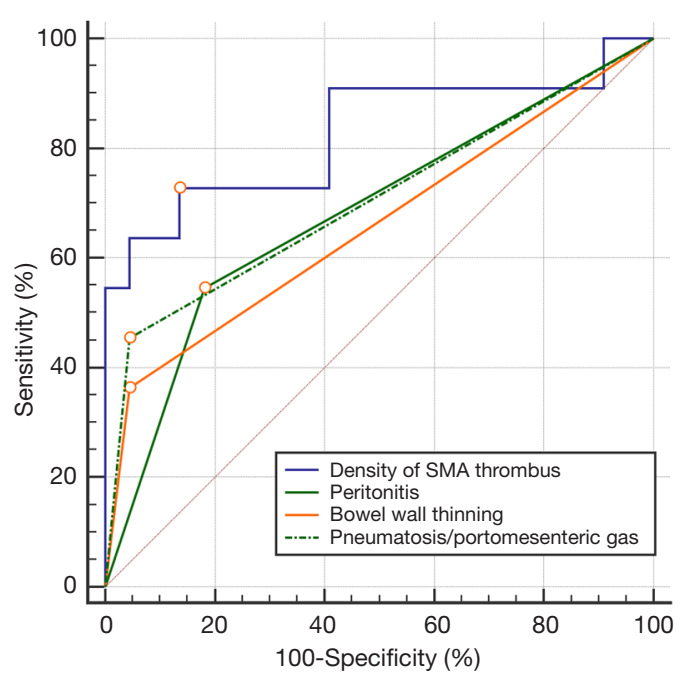

Figure 4 Receiver-operating characteristic (ROC) curve analyses of the superior mesenteric artery (SMA) thrombus density, peritonitis, bowel wall thinning, and pneumatosis/portomesenteric gas for diagnosing acute mesenteric ischemia (AMI) patients with transmural intestinal necrosis (TIN). The area under the ROC curve (AUC) of SMA thrombus density (0.83) was larger than those of peritonitis (0.68), bowel wall thinning (0.66), and pneumatosis/ portomesenteric gas (0.71), which suggested that SMA thrombus density may be more discriminative than the other three variables for predicting TIN.

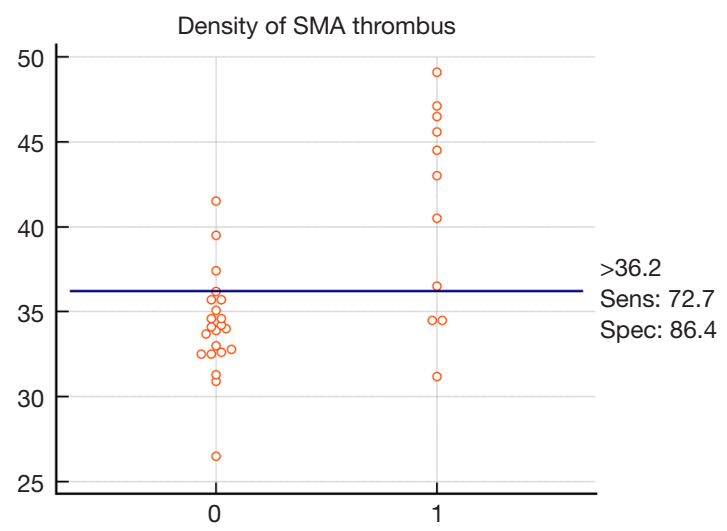

Figure 5 Interactive dot diagram of superior mesenteric artery (SMA) thrombus density [Hounsfield unit (HU)] with a cutoff value of 36.2. 0: acute mesenteric ischemia (AMI) without transmural intestinal necrosis (TIN); 1: AMI with TIN. Sens, sensitivity; Spec, specificity.

guide the selection of clinical treatment options. The present study was a retrospective analysis with a long time span, and the treatment options for these patients were decided according to the experiences of the attending physicians at that time. The patients underwent timely CT examination, and resection for suspected intestinal ischemic necrosis 
had be done within 24 hours following CT examination. To avoid bias associated with underestimation of intestinal necrosis, we excluded all patients with an interval of more than 24 hours between CT examination and resection.

The present study had some limitations that should be acknowledged. First, it was a retrospective analysis subject to selection bias. AMI patients with calcified SMA thrombi were excluded, as were those with thromboembolism only in SMA branches. Therefore, a degree of patient selection bias may have occurred. Second, our study had a small sample size; it was likely underpowered to detect the impact of certain risk factors on outcome. For instance, free intraperitoneal gas found on MDCT must be considered as an occurrence of TIN in patients with AMI. In our study, free intraperitoneal gas was found in only two patients with AMI on MDCT. The statistical results revealed that this MDCT sign was not associated with TIN; the incidence rate of free intraperitoneal gas was lower in patients with AMI. Third, we did not assess the volume or length of the SMA thrombus as risk factors for the occurrence of TIN. It is difficult to measure the volume and length of SMA thrombus on MDCT because of the curvature of the SMA. However, the degree of SMA trunk occlusion and the location and extent of SMA thrombus were not associated with TIN in our study, which indirectly indicates that the volume and length of SMA thrombus may not be related to TIN because of mesenteric circulation with extensive collateral circulation (34). Fourth, we analyzed the relationship of the mean density of the whole SMA thrombus with the occurrence of TIN in patients with AMI; however, the accuracy of application in clinical practice has yet to be verified by prospective, large-sample studies, including the relationship of the length or range of SMA thrombus with TIN. Finally, there was no pathological examination to confirm the composition of SMA thrombus.

In conclusion, the findings of the present study indicate that the SMA thrombus density, measured on admission using non-contrast MDCT images, may be a predictor of the risk of TIN in patients with AMI caused by SMA thromboembolism. A high SMA thrombus density on MDCT is independently associated with TIN, and a cutoff value of more than 36.2 HU may predict the occurrence of TIN in patients with AMI due to SMA thromboembolism. Larger prospective studies are needed to validate these findings.

\section{Acknowledgments}

Funding: This work was supported by the National Natural
Science Foundation of China (No. 81671943).

\section{Footnote}

Conflicts of Interest: All authors have completed the ICMJE uniform disclosure form (available at http://dx.doi. org/10.21037/qims-20-604). The authors have no conflicts of interest to declare.

Ethical Statement: The present retrospective study was approved by the Institutional Ethics Committee of Daping Hospital, which waived the requirement for written informed consent.

Open Access Statement: This is an Open Access article distributed in accordance with the Creative Commons Attribution-NonCommercial-NoDerivs 4.0 International License (CC BY-NC-ND 4.0), which permits the noncommercial replication and distribution of the article with the strict proviso that no changes or edits are made and the original work is properly cited (including links to both the formal publication through the relevant DOI and the license). See: https://creativecommons.org/licenses/by-nc-nd/4.0/.

\section{References}

1. Clair DG, Beach JM. Mesenteric ischemia. N Engl J Med 2016;374:959-68.

2. Ehlert BA. Acute gut ischemia. Surg Clin North Am 2018;98:995-1004.

3. Diamond SL. Systems analysis of thrombus formation. Circ Res 2016;118:1348-62.

4. Berndt M, Friedrich B, Maegerlein C, Moench S, Hedderich D, Lehm M, Zimmer C, Straeter A, Poppert H, Wunderlich S, Schirmer L, Oberdieck P, Kaesmacher $\mathrm{J}$, Boeckh-Behrens T. Thrombus permeability in admission computed tomographic imaging indicates stroke pathogenesis based on thrombus histology. Stroke 2018;49:2674-82.

5. Paterno F, Longo WE. The etiology and pathogenesis of vascular disorders of the intestine. Radiol Clin North Am 2008;46:877-85.

6. Chiu YW, Wu CS, Chen PC, Wei YC, Hsu LY, Wang SH. Risk of acute mesenteric ischemia in patients with diabetes: a population-based cohort study in Taiwan. Atherosclerosis 2020;296:18-24.

7. Wang X, Chu C, Sun S, Xie T, Duan Z, Wang K, Liu B, Fan X, Wu X, Ding W. Outcomes and clinical 
characteristics of transmural intestinal necrosis in acute mesenteric ischemia. Scand J Gastroenterol 2019;54:953-9.

8. Acosta S, Björck M. Modern treatment of acute mesenteric ischaemia. Br J Surg 2014;101:e100-8.

9. Kanasaki S, Furukawa A, Fumoto K, Hamanaka Y, Ota S, Hirose T, Inoue A, Shirakawa T, Hung Nguyen LD, Tulyeubai S. Acute mesenteric ischemia: multidetector CT findings and endovascular management. Radiographics 2018;38:945-61.

10. Mazzei MA, Guerrini S, Cioffi Squitieri N, Vindigni C, Imbriaco G, Gentili F, Berritto D, Mazzei FG, Grassi R, Volterrani L. Reperfusion in non-occlusive mesenteric ischaemia (NOMI): effectiveness of CT in an emergency setting. Br J Radiol 2016;89:20150956.

11. Mazzei MA, Guerrini S, Gentili F, Monteleone I, Lucii G, Mazzei FG, Volterrani L. Non-occlusive mesenteric ischemia as a fatal complication in acutepancreatitis: a brief radiological comment. Dig Dis Sci 2020;65:1553-5.

12. Nuzzo A, Maggiori L, Ronot M, Becq A, Plessier A, Gault N, Joly F, Castier Y, Vilgrain V, Paugam C, Panis Y, Bouhnik Y, Cazals-Hatem D, Corcos O. Predictive factors of intestinal necrosis in acute mesenteric ischemia: prospective study from an intestinal stroke center. Am J Gastroenterol 2017;112:597-605.

13. Klang E, Kerpel A, Soffer S, Zlotnik M, Shimon O, Rimon U, Konen E, Amitai MM. CT imaging features of symptomatic and asymptomatic floating aortic thrombus. Clin Radiol 2018;73:323.e9-14.

14. Schroeder S, Kopp AF, Burgstahler C. Noninvasive plaque imaging using multislice detector spiral computed tomography. Semin Thromb Hemost 2007;33:203-9.

15. Kirchhof K, Welzel T, Mecke C, Zoubaa S, Sartor K. Differentiation of white, mixed, and red thrombi: value of CT in estimation of the prognosis of thrombolysis phantom study. Radiology 2003;228:126-30.

16. Sandstrom CK, Ingraham CR, Monroe EJ, Johnson GE. Beyond decreased bowel enhancement: acute abnormalities of the mesenteric and portal vasculature. Abdom Imaging 2015;40:2977-92.

17. Corcos O, Castier Y, Sibert A, Gaujoux S, Ronot M, Joly F, et al. Effects of a multimodal management strategy for acute mesenteric ischemia on survival and intestinal failure. Clin Gastroenterol Hepatol 2013;11:158-65.e2.

18. Mazzei MA, Guerrini S, Lucii G, Mazzei FG, Volterrani L. Bowel obstruction and intestinal ischemia: warnings for radiologists. Abdom Radiol (NY) 2020;45:887-8.

19. Guerrini S, Bucalossi A, Cioffi Squitieri N, Mazzei FG, Volterrani L, Mazzei MA. Ischemic colitis diagnosed by magnetic resonance imaging during lenalidomide treatment in a patient with relapsed multiple myeloma. Tumori 2016. doi: 10.5301/tj.5000392.

20. Mazzei MA, Guerrini S, Cioffi Squitieri N, Imbriaco G, Mazzei FG, Volterrani L. Non-obstructive mesenteric ischemia after cardiovascular surgery: not so uncommon. Ann Thorac Cardiovasc Surg 2014;20:253-5.

21. Florim S, Almeida A, Rocha D, Portugal P. Acute mesenteric ischaemia: a pictorial review. Insights Imaging 2018;9:673-82.

22. Calame P, Malakhia A, Turco C, Grillet F, Piton G, Delabrousse E. Transmural bowel necrosis from acute mesenteric ischemia and strangulated small-bowel obstruction: distinctive CT features. AJR Am J Roentgenol 2020;214:90-5.

23. Jang KM, Min K, Kim MJ, Koh SH, Jeon EY, Kim IG, Choi D. Diagnostic performance of CT in the detection of intestinal ischemia associated with small-bowel obstruction using maximal attenuation of region of interest. AJR Am J Roentgenol 2010;194:957-63.

24. Yunoki K, Naruko T, Inoue T, Sugioka K, Inaba M, Iwasa Y, Komatsu R, Itoh A, Haze K, Yoshiyama M, Becker AE, Ueda M. Relationship of thrombus characteristics to the incidence of angiographically visible distal embolization in patients with ST-segment elevation myocardial infarction treated with thrombus aspiration. JACC Cardiovasc Interv 2013;6:377-85.

25. Jeong MJ, Kwon H, Noh M, Ko GY, Gwon DI, Lee JS, Kim MJ, Choi JY, Han Y, Kwon TW, Cho YP. Relationship of lower-extremity deep venous thrombosis density at CT venography to acute pulmonary embolism and the risk of postthrombotic syndrome. Radiology 2019;293:687-94.

26. Gersh KC, Nagaswami C, Weisel JW. Fibrin network structure and clot mechanical properties are altered by incorporation of erythrocytes. Thromb Haemost 2009;102:1169-75.

27. van Gelder JM, Nair CH, Dhall DP. The significance of red cell surface area to the permeability of fibrin network. Biorheology 1994;31:259-75.

28. Quadros AS, Cambruzzi E, Sebben J, David RB, Abelin A, Welter D, Sarmento-Leite R, Mehta RH, Gottschall CA, Lopes RD. Red versus white thrombi in patients with ST-elevation myocardial infarction undergoing primary percutaneous coronary intervention: clinical and angiographic outcomes. Am Heart J 2012;164:553-60.

29. Marchena-Gomez J, Acosta-Merida MA, HemmersbachMiller M, Conde-Martel A, Roque-Castellano C, 
Hernandez-Romero J. The age-adjusted Charlson

Comorbidity Index as an outcome predictor of patients with acute mesenteric ischemia. Ann Vasc Surg 2009;23:458-64.

30. Kärkkäinen JM, Acosta S. Acute mesenteric ischemia (part I) -Incidence, etiologies, and how to improve early diagnosis. Best Pract Res Clin Gastroenterol 2017;31:15-25.

31. Acosta S. Surgical management of peritonitis secondary to acute superior mesenteric artery occlusion. World J Gastroenterol 2014;20:9936-41.

Cite this article as: Tang W, Zhang J, Kuang LQ, Yi KM, Li CX, Wang Y. Relationship of superior mesenteric artery thrombus density with transmural intestinal necrosis on multidetector computed tomography in acute mesenteric ischemia. Quant Imaging Med Surg 2021;11(7):3120-3132. doi: 10.21037/qims-20-604
32. Olson MC, Fletcher JG, Nagpal P, Froemming AT, Khandelwal A. Mesenteric ischemia: what the radiologist needs to know. Cardiovasc Diagn Ther 2019;9:S74-87.

33. Segatto E, Mortelé KJ, Ji H, Wiesner W, Ros PR. Acute small bowel ischemia: CT imaging findings. Semin Ultrasound CT MR 2003;24:364-76.

34. van Petersen AS, Kolkman JJ, Meerwaldt R, Huisman AB, van der Palen J, Zeebregts CJ, Geelkerken RH. Mesenteric stenosis, collaterals, and compensatory blood flow. J Vasc Surg 2014;60:111-9, 119.e1-2. 